\title{
Transtorno do Desenvolvimento da Coordenação e Coocorrências: conhecendo o
}

\author{
"estado da arte" \\ Developmental Coordination Disorders and Cooccurring: knowing the "state of the art" \\ Trastorno del Desarrollo de la Coordinación y Comorbilidad: conocer el "estado del arte"
}

Recebido: 16/08/2021 | Revisado: 25/08/2021 | Aceito: 28/08/2021 | Publicado: 30/08/2021

\author{
Bruna Cecim de Souza \\ ORCID: https://orcid.org/0000-0001-5099-6625 \\ Universidade Federal do Amazonas, Brasil \\ E-mail: ccecimbruna@gmail.com \\ Geane das Chagas Silva \\ ORCID: https://orcid.org/0000-0002-1454-9964 \\ Universidade Federal do Amazonas, Brasil \\ E-mail: geanelayssa@gmail.com \\ Cleverton José Farias de Souza \\ ORCID: https://orcid.org/0000-0002-7085-6651 \\ Universidade Federal do Amazonas, Brasil \\ E-mail: cleverton@ufam.edu.br \\ Lúcio Fernandes Ferreira \\ ORCID: https://orcid.org/0000-0002-1193-6029 \\ Universidade Federal do Amazonas, Brasil \\ E-mail: lucciofer@ufam.edu.br
}

\begin{abstract}
Resumo
O objetivo do trabalho foi conhecer o estado da arte envolvendo o TDC e a coocorrência de outros Trasntornos Desenvolvimentais. Trata-se de estudo de revisão integrativa envolvendo as bases de dados: Sport Discus, Gale Academic, Google Scholar, Medline, Science Direct e Scopus, por meio do portal de periódicos da Coordenação de Aperfeicoamento de Pessoal de Nível Superior (CAPES). Os descritores utilizados foram: Developmental Coordination Disorder AND Co-occurrences AND Comorbidity; Developmental Disabilities AND Co-occurrences OR Comorbidity AND Developmental Coordination Disorder, no período de 2000 a 2020, na língua inglesa. A amostra final foi composta por 06 artigos e os resultados demonstraram que há poucas pesquisas relacionadas às coocorrências com o TDC; as fases desenvolvimentais presentes foram a infância e a adolescência e existem diversos testes que podem auxiliar na identificação dos transtornos desenvolvimentais. Concluímos que o tema em questão tem recebido pouca atenção dos estudiosos da área apesar da afirmação de que a coocorrência entre TDC e Transtornos Desenvolvimentais é frequente e não exceção.
\end{abstract}

Palavras-chave: Transtornos desenvolvimentais; Transtornos da habilidade motora; Coocorrências; Educação inclusiva; Evidências científicas.

\begin{abstract}
The objective of the work was to know the state of the art involving DCD and the co-occurrence of other Developmental Disorders. This is an integrative review study involving the following databases: Sport Discus, Gale Academic, Google Scholar, Medline, Science Direct and Scopus through the journal portal of the Coordination for the Improvement of Higher Education Personnel (CAPES). The descriptors used were: Developmental Coordination Disorder AND Co-occurrences AND Comorbidity; Developmental Disabilities AND Co-occurrences OR Comorbidity AND Developmental Coordination Disorder, from 2000 to 2020, in English. The final sample consisted of 06 articles and the results showed that there is little research related to co-occurrences with DCD; the developmental phases present were childhood and adolescence and there are several tests that can help to identify developmental disorders. We conclude that the subject in question has received little attention from scholars in the field despite the assertion that the co-occurrence between DCD and Developmental Disorders is frequent and not an exception.
\end{abstract}

Keywords: Developmental disorders; Motor skill disorders; Co-occurrences; Inclusive education; Scientifics evidences.

\section{Resumen}

El objetivo del trabajo fue conocer el estado del arte envolviendo el TDC y la comorbilidad de otros Trastornos Desarrollados. Se trata del estudio de revisión integrativo envolviendo las bases de datos: Sport Discus, Gale Academic, Google Scholar, Medline, Science Direct y Scopus por medio del portal de periódicos de la Coordinación 
de Perfección Personal de Nivel Superior (CAPES). Los descriptores utilizados fueron: Developmental Coordination Disorder AND Co-occurrences AND Comorbidity; Developmental Disabilities AND Co-occurrences OR Comorbidity AND Developmental Coordination Disorder en el periodo de 2000 a 2020, en la lengua inglesa. La muestra final fue compuesta por 06 artículos y los resultados demostraron que existen pocas investigaciones relacionas a las comorbilidades con el TDC; las presentes fases desarrolladas fueron en la infancia y en la adolescencia, y existen diversas pruebas que pueden auxiliar en la identificación de los trastornos desarrollados. Concluimos que el tema en cuestión ha recebido poca atención de los estudiosos del area apesar de la afirmación de que a comorbilidad entre TDC y Trastorno Desarrollados es frecuente y no excepción.

Palabras clave: Trastornos desarrollados; Trastornos de habilidade motora; Comorbilidad; Educación inclusiva; Evidencia científica.

\section{Introdução}

Transtornos desenvolvimentais, como o Transtorno do Desenvolvimento Intelectual (TDI), o Transtorno do Espectro Autista (TEA), o Transtorno Específico da Aprendizagem (TEA), o Transtorno da Comunicação (TC) e o Transtorno do Déficit de Atenção/Hiperatividade (TDAH) interferem negativamente no desempenho escolar, social e pessoal, causando limitações no domínio de funções executivas, na aprendizagem, nas habilidades sociais e motoras (APA). A esses, adicionamos o Transtorno do Desenvolvimento da Coordenação (TDC).

O TDC caracteriza-se por atrasos, prejuízos no desenvolvimento e aprendizagem de habilidades motoras que afetam as atividades da vida diária, como vestir-se, abotoar a camisa, escovar os dentes, pentear os cabelos (Missiuna, 2003); e atividades da vida escolar, a saber: escrever, desenhar, cortar com tesouras, ocasionando consequências nos relacionamentos sociais (Pulzi \& Rodrigues, 2015). Os impactos negativos são também sentidos nos domínios afetivo e social, bem como na saúde mental (Hill., 2011; Kirby et al., 2014; Zwicker et al., 2012) afetando a autoestima, a autoavaliação, o autoconceito e autocompetência (Losse et al., Missiuna, Gaines et al., 2006; Pike \& Kuh, 2005).

Sua prevalência na população infantil está estimada em 6\% (APA, 2014), no entanto, esse valor tem apresentado variações, às vezes para mais ou para menos, em diferentes países (Ferreira \& Freudenheim, 2017). Essas diferenças podem ser justificadas pelos diferentes processos de identificação; de seleção das amostras populacionais (Geuze et al., 2015; Lingam et al., 2009) e ainda, a forma como o TDC se manifesta e o ambiente em que está inserido.

A manifestação do TDC se dá nas diferentes classes de habilidades motoras, seja de forma específica, como nas habilidades manuais (escrever, arremessar a bola, receber, entre outras.), ou em duas, ou mais classes, como nas habilidades locomotoras e estabilizadoras (equilibrar-se em uma perna, saltitar, andar pela sala de aula, entre outras) (Ferreira \& Freudenheim, 2017).

Ele pode ocorrer isoladamente ou simultaneamente com outros transtornos desenvolvimentais, condição que chamamos de coocorrência (Kaplan, Crawford et al., 2006). A coocorrência não é algo isolado, ao contrário, pode ser muito mais presente do que possamos imaginar. O TDC pode coocorrer com o TDAH (Kaplan, Wilson et al., 1998; Piek, Pitcher, et., 1999), com a Dislexia (Alves et al., 2017; Geuze \& Kalverboer, 1994), com o Transtorno de Linguagem (Hill, 2001; Rintala et al., 1998), com a Incapacidade de Aprendizagem (Ehrhardt et al., 1987; Schaffer et al., 1989), entre outros.

Entendemos que o TDC é uma condição que merece atenção, pois além de ser um dos principais problemas de saúde entre crianças e adolescentes em idade escolar (Cairney et al., 2005; Hill \& Brown, 2013), também pode se constituir como um vetor de exclusão nos ambientes domésticos e educacionais, especialmente quando há coocorrência com outros transtornos. Nessa perspectiva, pretendemos conhecer o estado da arte envolvendo o TDC e a coocorrência de outros Transtornos Desenvolvimentais. 


\section{Metodologia}

Trata-se de estudo de revisão integrativa realizado em cinco etapas: (1) identificação do problema de pesquisa; (2) investigação bibliográfica sistematizada; (3) avaliação dos dados encontrados; (4) análise dos dados; e (5) apresentação das conclusões (Guerin et al., 2019; Whittemore \& Knafl, 2005). Na primeira etapa, elaboramos as seguintes questões norteadoras: a) Qual é o estado da arte científica com foco na coocorrência entre TDC e outros transtornos desenvolvimentais? b) Quais as coocorrências identificadas? c) Quais as fases desenvolvimentais envolvidas nos estudos? e d) Quais testes foram utilizados para identificar as coocorrências? Para responder tais questionamentos foram elaborados os seguintes objetivos: a) selecionar as produções científicas com foco na coocorrência entre TDC e outros transtornos desenvolvimentais; b) identificar as coocorrências entre o TDC e os transtornos desenvolvimentais; c) caracterizar as fases desenvolvimentais envolvidas nos estudos; e d) elencar os testes utilizados na identificação das coocorrências de transtornos desenvolvimentais.

A segunda etapa compreendeu as buscas dos artigos científicos com os descritores: (i) Developmental Coordination Disorder AND Co-occurrences AND Comorbidity; (ii) Developmental Disabilities AND Co-occurrences OR Comorbidity AND Developmental Coordination Disorder; nas seguintes bases de dados: Wile Online Library (WOL); Sport Discus (SD); Gale Academic (GA); Google Scholar (GS); Medline Complete (MC); Science Direct (Sc); e Scopus (Sp).

Nesta etapa utilizamos os seguintes critérios de elegibilidade: estudos publicados no formato de artigos completos revisados por pares na língua inglesa; estudos que abordassem às coocorrências de transtornos desenvolvimentais em indivíduos com o TDC de todas as faixas etárias; estudos que tratassem sobre a identificação das coocorrências e comorbidades de transtornos desenvolvimentais em indivíduos com TDC; e estudos produzidos entre os anos de 2000 a 2020. Os critérios de exclusão foram estudos de revisão; estudos de caso e estudos piloto (Figura 1).

\section{Resultados e Discussão}

Na busca inicial encontramos 1.716 artigos, dos quais 1.669 foram excluídos após a leitura dos títulos e resumos. Dos 47 artigos que seguiram para a próxima etapa, verificamos que 23 apresentavam duplicidade. Assim, 24 artigos foram lidos na íntegra e depois da verificação dos critérios de elegibilidade, 6 artigos compuseram nossa amostra final (Figura 1). 
Figura 1 - Busca dos artigos: segunda etapa.

\begin{tabular}{|c|c|c|c|c|c|c|}
\hline \multicolumn{6}{|c|}{$\begin{array}{l}\text { Total de artigos encontrados nas bases de dados } \\
\qquad \mathbf{1 . 7 1 6}\end{array}$} & \\
\hline $\begin{array}{c}\text { Wile Online } \\
\text { Library } \\
(\text { WOL }=184)\end{array}$ & $\begin{array}{l}\text { Scopus } \\
(\mathrm{Sp}=127)\end{array}$ & $\begin{array}{c}\text { Sport } \\
\text { Discus } \\
(\mathbf{S D}=19)\end{array}$ & $\begin{array}{c}\text { Science } \\
\text { Direct } \\
(\text { Sc }=301)\end{array}$ & $\begin{array}{l}\text { Medline } \\
\text { Complete } \\
(\mathrm{MC}=153)\end{array}$ & $\begin{array}{l}\text { Google } \\
\text { Scholar } \\
(\text { GS=95) }\end{array}$ & $\begin{array}{c}\text { Gale } \\
\text { Academic } \\
(\mathbf{G A}=\mathbf{8 3 7})\end{array}$ \\
\hline
\end{tabular}

Total de artigos excluídos devido ao critério de elegibilidade

1.669

Total de artigos que permaneceram

47

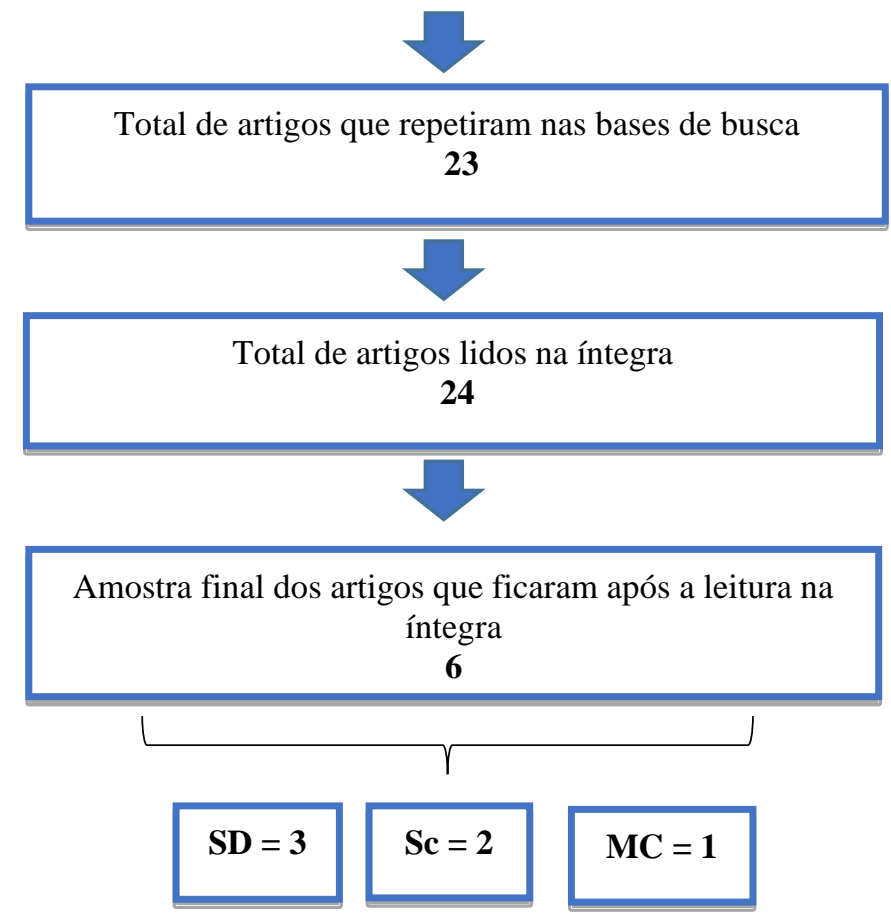

Fonte: Autores (2021).

A seguir, discutimos nossos resultados seguindo a ordem de nossas perguntas norteadoras em atendimento às etapas três e quatro de nossa metodologia.

\subsection{Produções cientificas com foco na coocorrência entre TDC e outros Transtornos Desenvolvimentais}

A coocorrência entre TDC e outros Transtornos Desenvolvimentais parece ser mais comum do que exceção (Kaplan, Wilson et al., 1998), isto significa que, a maioria dos indivíduos com TDC tendem a apresentar outros transtornos concomitantemente, tais como as dificuldades específicas de aprendizado, como a Dislexia e o Comprometimento Específico da Linguagem, e Problemas de Atenção e Concentração (Visser, 2003) entre outros. Como nos mostra os estudos relacionados 
no Quadro 1.

Quadro 1 - Relação de estudos com foco na coocorrência entre TDC e outros transtornos.

\begin{tabular}{|c|c|l|l|}
\hline $\mathbf{N}^{\mathbf{0}}$ & Base & \multicolumn{1}{|c|}{ Título } & \multicolumn{1}{|c|}{ Autores e ano } \\
\hline 1 & $\mathrm{MC}$ & Comorbidity, co-occurrence, continuum: what's in a name? & Kaplan, Crawford, et al. (2006). \\
\hline 2 & $\mathrm{SD}$ & $\begin{array}{l}\text { Co-occurring disorders: A possible Key to visual perceptual deficits in } \\
\text { children with developmental coordination disorder? }\end{array}$ & Crawford e Dewey (2008). \\
\hline 3 & Sc & $\begin{array}{l}\text { Comorbidity of motor and language impairments in preschool children } \\
\text { of Taiwan }\end{array}$ & Cheng et al. (2009). \\
\hline 4 & SD & $\begin{array}{l}\text { An examination of the relationship between movement problems and } \\
\text { four common developmental disorders }\end{array}$ & Martin et al. (2010). \\
\hline 5 & Sc & $\begin{array}{l}\text { Developmental Coordination Disorder in children with specific } \\
\text { language impairment: Co-morbidity and impact on quality of live }\end{array}$ & Flapper e Scoemaker (2013). \\
\hline 6 & SD & $\begin{array}{l}\text { Reading and Writing Skills in Children with Specific Learning } \\
\text { Disabilities With and Without Developmental Coordination Disorder }\end{array}$ & Di Brina et al. (2018). \\
\hline
\end{tabular}

Fonte: Autores.

Percebemos que temática envolvendo a coocorrência entre o TDC e os Transtornos Desenvolvimentais recebeu pouca atenção nos últimos 20 anos, período no qual, somente nove artigos foram produzidos. Esse panorama já era apontado por Visser (2003) ao afirmar que embora cientes da existência da coocorrência entre esses transtornos, os pesquisadores não direcionavam seus esforços em investigá-la. Isso nos parece um contrassenso, pois, sendo a coocorrência algo frequente, não pode ser tratada como exceção (Caçola \& Lage, 2019).

Dessa maneira, concordamos Ferreira e Freudenhim (2017), de que essa temática deve ser considerada pelos pesquisadores, principalmente por envolver as fases desenvolvimentais da infância e adolescência que correspondem a todo o período escolar da educação básica, compondo uma etapa importante no desenvolvimento humano (Papalia \& Feldman, 2013). Investigar a coocorrência entre TDC e outros Transtornos Desenvolvimentais pode revelar as necessidades educativas especiais (NEE) de crianças e adolescentes e permitir que recebam suporte necessário à sua inclusão nas atividades cotidianas e escolares.

As NEE são abrangentes e incorporam os grupos com dificuldades de aprendizagem por diversas origens como os que apresentam Transtornos Desenvolvimentais e, em especial, aqueles com TDC e transtornos coocorrentes. $\mathrm{O}$ atendimento às NEE possibilita a presença garantida no contexto escolar inclusivo pautado em projetos políticos pedagógicos flexíveis e dinâmicos, sem dar vez a exclusão (Fernandes, 2013).

A escola, que segue os princípios da educação inclusiva, adota uma política que direciona suas ações por meio da valorização da participação ativa do aluno, através de práticas voltadas aos conhecimentos construídos e partilhados (Rodrigues, 2006), oportunizando o acesso, participação e aprendizagem via práticas pedagógicas que valorizem a diferença e promovam o desenvolvimento de todos (Brasil, 2008). Para que isso se concretize é fundamental o conhecimento e o atendimento às NEE de crianças e adolescentes com os Transtornos Desenvolvimentais, notadamente, daqueles que já apresentem o TDC. Outra ação importante é a identificação das coocorrências.

\subsection{Identificação das coocorrências}

Atendendo ao nosso segundo (b) objetivo, apresentamos as coocorrências identificadas nos artigos analisados (Quadro 
2).

Quadro 2 - Identificação das coocorrências entre o TDC e os transtornos desenvolvimentais.

\begin{tabular}{|c|c|c|}
\hline $\mathbf{N}^{\mathbf{o}}$ & Uma coocorrência com o TDC & Duas coocorrências com o TDC \\
\hline 1 & $\begin{array}{l}\text { Transtorno do Déficit de Atenção com Hiperatividade } \\
\text { (TDAH). }\end{array}$ & $\begin{array}{l}\text { Transtorno do Déficit de Atenção com Hiperatividade } \\
\text { (TDAH), mais a Dificuldade de Leitura (DL). }\end{array}$ \\
\hline 2 & $\begin{array}{l}\text { Transtorno do Déficit de Atenção com Hiperatividade } \\
\text { (TDAH); Dificuldade de Leitura (DL). }\end{array}$ & $\begin{array}{l}\text { Transtorno do Déficit de Atenção com Hiperatividade } \\
\text { (TDAH), mais a Dificuldade de Leitura (DL). }\end{array}$ \\
\hline 3 & Transtorno do Desenvolvimento da Fala e Linguagem (TDL). & \\
\hline 4 & Dificuldade de Leitura (DL). & $\begin{array}{l}\text { Transtorno do Déficit de atenção com Hiperatividade } \\
\text { (TDAH), mais o Transtorno Desafiador de Oposição (TOD), } \\
\text { mais o Dificuldade de Leitura (DL). }\end{array}$ \\
\hline 5 & Distúrbio Específico de Linguagem (DEL). & \\
\hline 6 & Dificuldade de Aprendizagem (DA). & \\
\hline
\end{tabular}

Fonte: Autores.

Observamos uma heterogeneidade nos transtornos desenvolvimentais que coocorrem com o TDC. Verificamos também que, há casos que apresentam mais de um Transtorno Desenvolvimental, o que não é incomum (Kaplan, Wilson et al., 1998). Dentre os transtornos identificados, os mais frequentes foram o Transtorno do Déficit da Atenção e Hiperatividade (TDAH) (Crawford \& Dewey, 2008; Kaplan, Crawford et al., 2006; Martin et al., 2010) e a Dificuldade de Aprendizagem (DA) (Crawford \& Dewey, 2008; Di Brina et al., 2018; Kaplan, Crawford et al., 2006; Martin et al., 2010) que envolve dificuldades na leitura, na escrita e na matemática (APA, 2014).

As estimativas da coocorrência entre o TDC e o TDAH está na ordem de 50\% dos casos (APA, 2014; Martin et al., 2010). Embora alguns aspectos relativos a essa coocorrência permaneçam incertos, pois, ora as dificuldades motoras apresentadas no TDAH são provenientes da desatenção e da impulsividade, ora são consideradas como uma consequência da coocorrência com o TDC (Goulardins, 2016).

No TDC pode ocorrer o baixo desempenho nas habilidades motoras e consequentemente dificuldades para escrever, se organizar, planejar e modificar movimentos durante as atividades realizadas. Contudo, é importante lembrar que as características do TDC são diversas (Villa et al., 2020). Tal como quando há coocorrência entre o TDC e o TDAH as consequências podem ser mais severas do que quando esses transtornos se manifestam isoladamente (Goulardins et al., 2015).

As Dificuldades de Aprendizagem ocorrem em 5\% a 10\% da população e estão relacionadas ao mau desempenho na leitura, escrita e na matemática podendo interferir significativamente no rendimento escolar e nas atividades do cotidiano de crianças e adolescentes que exigem habilidades nessas competências escolares, com o agravante de acompanharem o indivíduo em todas as fases do seu ciclo vital (Siqueira \& Giannetti, 2011).

As dificuldades na leitura ocorrem quando o desempenho nessa habilidade (associação de letra-som, habilidade de ler palavras e proficiência na leitura) é inferior ao esperado para a idade em fase escolar, ocasionando graves consequências educacionais, sociais e emocionais (Rotta, 2006; Siqueira \& Giannetti, 2011). Para as dificuldades observadas na escrita temos letras ilegíveis e incompletas, dificuldade para associar letra e som, e dificuldades espaciais (Ciasca, 2003); as dificuldades na 
matemática são identificadas em somas simples, problemas com sinais operacionais, dificuldades em estruturar a conta, ler números com multidígitos (Bastos, 2007).

A coocorrência entre TDC e a DA pode resultar em dificuldades gravíssimas para crianças e adolescente, pois o TDC pode, também, manifestar-se na escrita e na organização espacial (Siqueira \& Giannetti, 2011). Essas dificuldades podem gerar sentimentos de incapacidade, insucesso e fracasso que, por sua vez, podem conduzir crianças e adolescentes a se afastarem do ambiente escolar. Por outro lado, diferentemente do TDAH, até o presente momento, não temos estimativas da coocorrência entre o TDC e a DA, no entanto, supomos que sua frequência seja semelhante ou superior ao do TDAH e que a carência de estudos subdimensione esses valores.

Outros Transtornos Desenvolvimentais identificados foram o Transtorno do Desenvolvimento da Fala e Linguagem (Cheng et al., 2009) e o Distúrbio Específico de Linguagem (Flapper \& Schoemaker, 2013). Os transtornos da comunicação incluem déficit na linguagem, na fala e na comunicação. As características centrais do Transtorno da Linguagem são as dificuldades na aquisição e no uso da linguagem, déficit na compreensão ou na produção de vocabulários, na estrutura das frases e no discurso (APA, 2014).

O Transtorno da Fala se caracteriza por dificuldades fonológicas de sons da fala que combinados formam as palavras faladas, e o Transtorno de Comunicação pela dificuldade na comunicação social, resultando em limitações funcionais na participação, no desenvolvimento de relações sociais, no sucesso escolar ou no desempenho profissional. Além disso, o Transtorno específico da linguagem não está associado a comunicação verbal anormal e nem a presença de comportamentos restritos e/ou repetitivos (APA, 2014).

A identificação precoce dos transtornos que coocorrem com o TDC é um processo crucial para que medidas auxiliares sejam elaboradas e aplicadas com o intuito de que crianças e adolescentes possam desenvolver recursos próprios para enfrentar e superar as demandas ambientais. Dessa forma, acreditamos ser válido caracterizar as fases desenvolvimentais envolvidas nos estudos de nossa revisão.

\subsection{Caracterização das fases desenvolvimentais envolvidas nos estudos}

As fases desenvolvimentais envolvidas nos estudos foram a infância - 4 à 10 anos de idade - (Cheng et al., 2009; Di Brina et al., 2018; Flapper \& Schoemaker, 2013; Kaplan, Crawford et al., 2006) e a adolescência - 11 à 17 anos de idade (Crawford \& Dewey, 2008; Martin et al., 2010). Isso não nos surpreende, afinal, são fases sensíveis e que podem influenciar, negativa ou positivamente, as fases desenvolvimentais posteriores.

$\mathrm{Na}$ infância, especialmente na idade escolar, crianças com TDC podem apresentar dificuldades em lidar com as exigências ambientais, isto é, com atividades escolares típicas como escrever, recortar, pintar, participar de jogos e brincadeiras do cotidiano infantil (Ferreira \& Freudenheim, 2017; Missiuna, 2003; Papalia \& Feldman, 2013). A manifestação do transtorno motor, isoladamente, já é suficiente para interferir negativamente no processo desenvolvimental (Henderson \& Hall, 1982) o que esperar então, quando há coocorrência de outros Transtornos Desenvolvimentais, como o TDAH e a DA? Essa questão nos mostra a necessidade da identificação, avaliação e intervenção para melhor atendimento das NEE que emanam do TDC e dos demais Transtornos Desenvolvimentais, e que permita à criança ajustar seus recursos para o enfrentamento de novas demandas na adolescência.

A adolescência caracteriza-se como uma fase de instabilidade hormonal e sentimental, pela busca por aceitação e pelo sentimento de pertencimento social, isto é, as opiniões do ciclo familiar cedem lugar às do ciclo de amigos (Carvajal, 1998; Papalia \& Feldman, 2013). No entanto, adolescentes com dificuldades de aprendizagem são considerados menos populares, menos cooperativos, são rejeitados pelos seus colegas de classe e, tendem a manifestar problemas de comportamento (Kavale \& Forness, 1996). Somando-se a isso, podemos ter o isolamento social, o sentimento de frustração, a rejeição e o medo de 
realizar atividades em grupo (Hill, 2011; Tal-saban et al., 2012). Na esteira dessas manifestações teremos, ainda, adolescentes que podem apresentar baixa percepção de suas competências escolares e motoras (Schoemaker \& Kalverboer, 1994), passando por experiências de fracasso e frustração, além da exclusão de importantes atividades do cotidiano escolar. Provavelmente serão alvos de comentários pejorativos e de bullying tanto de colegas quanto de professores (Bejerot et al., 2011; Ferreira et al., 2015).

Concordamos que a melhor forma de auxiliá-los passa pelo conhecimento, a identificação do TDC e da coocorrência de Transtornos Desenvolvimentais e a compreensão das NEE de crianças e adolescentes para que possamos elaborar intervenções que melhorem seus recursos no enfrentamento das diferentes demandas do ambiente doméstico, escolar e social (Missiuna, Moll et al., 2007). Para isso, a utilização de testes no processo de identificação tem grande importância.

\subsection{Testes utilizados na identificação das coocorrências de transtornos desenvolvimentais em indivíduos com TDC}

A utilização de testes em pesquisas requer que os mesmos tenham medidas válidas, isto é, que meçam aquilo que eles se propõem a medirem (Thomas \& Nelson, 2002). Elencamos quinze testes utilizados nos artigos selecionados que mediram a coocorrência de diferentes Transtornos Desenvolvimentais em crianças e adolescentes com TDC (Quadro 3).

Quadro 3 - Testes utilizados para identificação das coocorrências.

\begin{tabular}{|c|c|c|}
\hline Coocorrências & Instrumentos & Autores dos instrumentos \\
\hline $\begin{array}{l}\text { Transtorno de Déficit } \\
\text { de Atenção e e } \\
\text { Hiperatividade. }\end{array}$ & $\begin{array}{l}\text { 1)Child Behaviour Checklist-CBCL; } \\
\text { 2)Parent Diagnostic Interview Schedule for Children-DISC; } \\
\text { 3)Abbreviated Symptom Questionnaire. }\end{array}$ & $\begin{array}{l}\text { 1) Achenbach (1991); } \\
\text { 2) Costello et al. (1985); } \\
\text { 3) Goyette et al. (1978). }\end{array}$ \\
\hline $\begin{array}{l}\text { Transtorno Desafiador } \\
\text { de Oposição. }\end{array}$ & 4)Australian twin behaviour rating scale (ATBRS). & 4) Levy e Hay (2001). \\
\hline Dificuldade de Leitura. & $\begin{array}{l}\text { 1)Woodcock Johnson Psychoeducational Battery-Revised-WJ-R; } \\
\text { 2)subteste Spelling do teste chamado A Wide Range Achievement } \\
\text { Test-Revised; } \\
\text { 3) Auditory Analsysis Test; } \\
\text { 4)Reading Disability Questionnaire (RDQ). }\end{array}$ & $\begin{array}{l}\text { 1) Woodcock e Johnson (1989); } \\
\text { 2) Jastak e Wilkinson (1984); } \\
\text { 3) Rosner e Simon (1971); } \\
\text { 4) Willcutt et al. (2011). }\end{array}$ \\
\hline $\begin{array}{ll}\text { Transtorno } & \text { do } \\
\text { Desenvolvimento } & \text { da } \\
\text { Fala e Linguagem. } & \end{array}$ & $\begin{array}{l}\text { 1)Peabody Picture Vocabulary Test-Revised-PPVT-R; } \\
\text { 2)The Language Ability Assessment for Preschoolers; } \\
\text { 3)Composite Speech/Language Test. }\end{array}$ & $\begin{array}{l}\text { 1) Lu e Liu (2005); } \\
\text { 2) Chang (1991); } \\
\text { 3) Lin (1992); Lin e Lin (1996). }\end{array}$ \\
\hline $\begin{array}{l}\text { Distúrbio Específico de } \\
\text { Linguagem. }\end{array}$ & $\begin{array}{l}\text { 1)Dutch Language Test for Children; } \\
\text { 2)Reynell Developmental Languagem Scales. }\end{array}$ & $\begin{array}{l}\text { 1) Bon (1982); } \\
\text { 2)Eldik et al. (1997). }\end{array}$ \\
\hline $\begin{array}{l}\text { Dificuldade } \\
\text { Aprendizagem. }\end{array}$ & $\begin{array}{l}\text { 1)Battery for the Evaluation of Developmental Dyslexia and } \\
\text { Dysorthographia (DDE-2); } \\
\text { 2)The Concise Assesment Method for Children Handweiting } \\
(B H K) \text {. }\end{array}$ & $\begin{array}{l}\text { 1)Sartori et al. (2007); } \\
\text { 2)Hamstra-bletz et al. (1987). }\end{array}$ \\
\hline
\end{tabular}


Para verificar a coocorrência do TDAH encontramos quatro testes (Crawford \& Dewey, 2008; Kaplan, Crawford et al., 2006; Martin et al., 2010), o Child Bevahiour Checklist (Achenbach, 1991) que é utilizado para identificar problemas comportamentais e emocionais em crianças e adolescentes com versões aplicáveis para os pais e/ou responsáveis, para crianças e adolescentes, e para os professores. As suas versões brasileiras são oficiais, mas estudos de validação ainda são necessários para que sejam utilizados (Bordin et al., 2013). O Parent Diagnostic Interview Schedule for Children (Costello et al., 1985) é um instrumento de diagnóstico totalmente estruturado, projetado e formulado com base no DSM-IV e avalia a presença ou ausência de 30 transtornos em crianças e adolescentes. Profissionais da área da saúde e pesquisadores podem utilizá-lo. O Abbreviated Symptom Questionnaire (Goyette et al., 1978) é utilizado por professores e possui 12 questões com 4 opções de respostas. O questionário é aplicável em crianças de 4 a 12 anos de idade. O quarto teste elencado é o Australian Twin Behaviour Rating Scale (Levy \& Hay, 2001) questionário que visa identificar sintomas do TDAH e também do Transtorno Desafiador de Oposição, envolve crianças de 6 a 9 anos de idade e deve ser respondido pelos pais e responsáveis.

Para a coocorrência de Dificuldade de Leitura identificamos outros quatro testes (Crawford \& Dewey, 2008; Kaplan, Crawford et al., 2006; Martin et al., 2010). O primeiro, Woodcock Johnson Psychoeducational Battery-Revised (Woodcock \& Johnson, 1989) foi elaborado para medir habilidades cognitivas; realizações escolares; e atitudes escolares, sua aplicação pode ser feita de forma individual ou coletiva em crianças de 5 a 9 anos. O segundo é o Wide Range Achievement Test-Revised (Jastak \& Wilkinson, 1984) que mede habilidades de leitura, matemática, ortografia e compreensão. Pode ser usado como avaliação inicial de desempenho escolar, reavaliação ou como medida de progresso dos alunos de 5 a 10 anos de idade. A seguir temos o Auditory Analysis Test (Rosner \& Simon 1971; Rosner, 1993) que avalia a capacidade das crianças entre 6 a 11 anos de idade. Este instrumento identifica os sons separados em palavras faladas e sequências de sons. O quarto teste é o Reading Disability Questionnaire (Willcutt et al., 2011), trata-se de um questionário aplicável aos pais e/ou responsáveis que avalia distúrbios de leitura de crianças e adolescentes de 5 a 16 anos.

A coocorrência do Transtorno do Desenvolvimento da Fala e Linguagem foi verificada por meio de três testes (Cheng et al., 2009). O primeiro é o Peabody Picture Vocabulary Test-Revised (Lu \& Liu, 2005) mede o vocabulário auditivo e fornece uma estimativa rápida da habilidade verbal, consiste em 175 itens de vocabulário de dificuldade e é destinado a indivíduos entre 3 e 11 anos de idade. O segundo teste é o Language Ability Assessment for Preschoolers (Chang, 1991) que mede a capacidade de linguagem e de compreensão de sentenças receptivas de crianças entre 4 e 6 anos. O terceiro é o Composite Speech/Language Test (Lin, 1992; Lin \& Lin, 1996) que é composto de cinco subtestes: articulação, pronúncia, fluência, linguagem receptiva, e linguagem expressiva, aplicável para as idades de 3 a 15 anos de idade.

$\mathrm{Na}$ identificação da coocorrência do Distúrbio Específico de Linguagem observamos dois testes (Flapper \& Schoemaker, 2013). O Dutch Language Test for Children (Bon, 1982), composto por 16 subtestes que medem habilidades de sintaxe, semântica, linguagem expressiva e receptiva em crianças de 4 a 10 anos de idade e o Reynell Developmental Languagem Scales (Eldik et al., 1997) que verifica habilidades linguísticas receptivas, a compreensão verbal e a linguagem expressiva em crianças de 1 ano e 3 meses a 7 anos e 6 meses.

Por fim, temos dois testes (Di Brina et al., 2018) que verificaram a coocorrência de Dificuldade de Aprendizagem. A Battery for the Evaluation of Developmental Dyslexia and Dysorthography (Sartori, Job \& Tressoldi, 2007) composta de oito subtestes, cinco para a análise do processo de leitura (nomeação de grafemas, leitura de palavras e não palavras, compreensão de frases com homófonos e correção de homófonos); e três para a análise do processo de escrita (ditado de palavras/não palavras e ditado de frases com homofônicas), aplicável em crianças de 7 a 14 anos. Por fim, temos o The Concise Assesment Method for Children Handwriting (Hamstra-Bletz et al., 1987) consiste em avaliar a legibilidade da escrita em crianças de 7 a 12 anos. A sua avaliação mede, mais especificamente, a qualidade em características morfológicas e espaciais da escrita, a velocidade e o número de letras escritas. 
Ressaltamos que, os testes são ferramentas necessárias para a identificação de Transtornos Desenvolvimentais coocorrentes ao TDC, no entanto, a presença de uma equipe multiprofissional é fundamental para apoiar e tornar o processo de avaliação o mais fidedigno possível.

\section{Conclusão}

Concluímos que o tema em questão tem recebido pouca atenção dos estudiosos da área apesar da afirmação de que a coocorrência entre TDC e Transtornos Desenvolvimentais é frequente e não exceção; é imperativo aumentar o volume de estudos envolvendo as diferentes fases desenvolvimentais; fortalecer as evidências científicas sobre os diferentes transtornos que podem coocorrer; e utilizar testes que meçam com segurança aquilo que se propõem a medir, ação crucial na identificação dos Transtornos Desenvolvimentais coocorrentes.

Crianças e adolescentes identificados com Transtornos Desenvolvimentais requerem ações e atitudes adequadas às suas NEE. Quanto mais precoce for a identificação, maiores serão as possibilidades de auxílio, organização, preparação e intervenção tornando a criança e o adolescente aptos ao enfrentamento das diferentes demandas ambientais e o contexto verdadeiramente inclusivo.

\section{Agradecimentos}

Agradecemos à Coordenação de Aperfeiçoamento de Pessoal de Nível Superior (CAPES); à Fundação de Amparo à Pesquisa do Estado do Amazonas (FAPEAM), a Universidade Federal do Amazonas (UFAM) e ao Programa de PósGraduação em Educação (PPGE) da Universidade Federal do Amazonas (FACED/UFAM) pelo apoio financeiro recebido.

\section{Referências}

Achenbach, T. M. (1991). Child Behavior Checklist for age 4-18. Parent Form. University of Vermont, Burlington, VT, USA.

Alves, J. F. M., Siqueira, C. M., Lodi, D. F., Aguiar, J. F. D., Mangelli, M. C., \& Alves, L. M. (2017). Dislexia e Transtorno do Desenvolvimento da Coordenação: ocorrência e coocorrência em um centro diagnóstico. Revista $N B C, 7(13), 1-13$.

American Psychiatric Association (APA) (2014). Diagnostic and statistical manual of mental disorders. (5a ed.).

Bastos, J. A. (2007). O cérebro e a matemática. Edição do autor.

Bejerot, S., Edgar, J., \& Humble, M. B. (2011). Poor performance in physical education - a risk factor for bully victimization: A case-control study. Acta Paediatrica, 100, 413-419. https://pubmed.ncbi.nlm.nih.gov/21039827/

Bon, V. W. H. J. (1982). Taaltest voor kinderen Lisse, The Netherlands: Swets \& Zeitlinger.

Bordin, I. A., Rocha, M. M., Paula, C. S., Teixeira, M. C. T. V., Achenbach, T. M., \& Rescorla, L. A. (2013). Child Behavior Checklist (CBCL), Youth SelfReport (YRS) and Teacher's Report Form (TRF): an overview of the developmental of the original and Brazilian versions, Cad. Saúde Pública, 29(1) 13-28. https://doi.org/10.1590/S0102-311X2013000100004

Brasil. (2008). Ministério da Educação. Política Nacional de Educação Especial na Perspectiva da Educação Inclusiva.

Caçola, P., \& Lage, G. (2019). Transtorno de Coordenação do Desenvolvimento (DCD): Uma visão geral da condição e evidências de pesquisa. Motriz: Revista de Educação Física, 25(2), 1-12.

Cairney, J., Hay, J. A., Faught, B. E., \& Hawes, R. (2005). Developmental Coordination Disorder and overweight and obesity in children aged 9-14 y. International Journal of Obesity, 29, 369-372. https://www.nature.com/articles/0802893

Carvajal, G. (1998). Torna-se Adolescente - a Aventura de uma Metamorfose. Ed. Cortez.

Chang, H. W. (1991). Language ability assessment for preschoolers (National Science Committee 79-0301-H-002-067).: Taiwan Government Printing Office.

Cheng, H. C., Chen, H. Y., Tsai, C. L., Chen, Y. J., \& Cherng, R. J. (2009). Comorbidity of motor and language impairments in preschool children of Taiwan. Revista Research in Developmental Disabilities, 30(5), 1054-1061, 2009.

Ciasca S. M. (2003). Distúrbios e dificuldades de aprendizagem: questão de nomenclatura. In: S. M. Ciasca (Org.), Distúrbios de aprendizagem: proposta de avaliação interdisciplinar. (3a ed.). São Paulo: Casa do Psicólogo. 
Costello, E. J., Edelbrock, C. S., \& Costello, A. J. (1985). Validity of the NIMH Diagnostic Interview Schedule for Children: a comparison between psychiatric and pediatric referrals. Journal of Abnormal Child Psychology, 13, 579-595. https://pubmed.ncbi.nlm.nih.gov/4078188/

Crawford, S. G., \& Dewey, D. (2008). Co-occurring disorders: A possible Key to visual perceptual deficits in children with developmental coordination disorder? Revista Human Movement Science, 27(1), 154-169. https://doi.org/10.1016/j.humov.2007.09.002

Di Brina, C., Averna, R., Rampoldi, P., Rossetti, S., \& Penge, R. (2018). Reading and Writing skills in children with specific learning disabilities with and without developmental coordination disorder. Revista Motor Control, 22(4), 391-405. https://doi.org/10.1123/mc.2016-0006

Ehrhardt, P., Mckinlay, I. A., \& Bradley, G. (1987). Coordination Screening For Children With And Without Moderate Learning Difficulties: Further Experience With Gubbay'S Test. Developmental Medicine \& Child Neurology, 29(5), 666-673.

Eldik, M. V., Schlichting, J. E. T. P., Spelberg, H. C. L., Vander-Meulen, B. F., \& Vander-Meulen S. J. (1997). Reynell Test voor Taalbegrip. (2e.). druk. Berkhout, Nijmegen.

Fernandes, S. (2013). Fundamentos para educação especial. Inter saberes.

Ferreira, L. F., Cabral, G. C. F., Santos, J. O. L., Souza, C. J. F., \& Freudenheim, A. M. (2015). Transtorno do Desenvolvimento da Coordenação: Discussões iniciais sobre programas de intervenção. Revista Acta Brasileira de Movimento Humano, 5(1), $42-65$.

Ferreira, L. F., \& Freudenheim, A. M. (2017). Noções desenvolvimentais e o transtorno do desenvolvimento da coordenação. Ed. CRV Ltda.

Flapper, B., \& Schoemaker, M. (2013). Developmental Coordination Disorder in children with specific language impairment: Co-morbidity and impact on quality of live. Revista research in Developmental Disabilities, 34(2), 756-763.

Geuze, R. H., \& Kalverboer, A. V. (1994). Tapping a rhythm: a problem of timing for children who are clumsy and dyslexic. Adapted Physical Activity Qarterly, 11, 203-213.

Geuze, R. H., Schoemaker, M. M., \& Smits-Englesman, B. C. M. (2015). Clinical and Research Criteria for Developmental Coordination Disorder - Should They Be One and the Same? Current developmental disorders reports, 2(2), 127-130.

Goulardins, J. B., Rigoli, D., Licari, M., Piek, J. P., Hause, R., Oosterlaan, J., \& Oliveira, J. A. (2015). Attention deficit hyperactivity disorder and developmental coordination disorder: Two separate disorder or do they share a common etiology. Behavioral Brain Research, 292, 484-492. https://doi.org/10.1016/j.bbr.2015.07.009

Goulardins, J. B. (2016). Desempenho motor de crianças com transtorno do déficit de atenção e hiperatividade e transtorno do desenvolvimento da coordenação [Tese de Doutorado, Escola de Educação Física e Esporte da Universidade de São Paulo].

Goyette, C. H., Conners, C. K., \& Ulrich, R. F. (1978). Normative data on revised Conners parent and teacher rating scales. Journal of Abnormal Child Psychology, 6, 221-236.

Guerin, C. S., Grehs, B. M. S., \& Coutinho, C. (2019). Estratégias educativas e recursos pedagógicos para o ensino e aprendizagem de alunos com TDAH: Uma revisão integrativa. Revista Valore, 4(1), 923-935.

Hamstra-Bletz, L., De-Bie, J., \& Den-Brinker, B. P. L. M. (1987). Beknopte beoordelingmethodevoor kinderhandschiften [The concise assessment Scale for children's handwriting]. Lisse, The Netherlands: Swets \& Zeitlinger.

Henderson, S. E. \& Hall, D. (1982). Concomitants of Clumsiness in Young Schoolchildren. Developmental Medicine \& Child Neurology, 24(5), 448-460.

Hill, E. L., Brown, D., \& Sorgardt, K. S. (2011). A preliminary Investigation of quality of live satisfaction reports in emerging adults with and without Developmental Coordination Disorder. Journal Adult Dev.

Hill, E. L., \& Brown, D. (2013). Mood impairments in adults previously diagnosed with developmental coordination disorder. Journal of Mental Health, 22(4), 334-340.

Hill, E. L. (2001). Non-Specific nature of specific language impairment: a review of the literature with regard to concomitant motor impairments. International Journal of Language \& Comunication Disorders, 36(2), 149-171.

Kavale, K. A., \& Forness, S. R. (1996). Social Skill Deficits and Learning Disabilities: A Meta-Analysis. Journal of Learning Disabilities, $29(3), 226-237$.

Kaplan, B., Crawford, S., Cantell, M., Kooistra, L., \& Dewey, D. (2006). Comorbidity, co-occurrence, continuum: what's in a name? Revista Child: care, health and developmental, 32(6), 723-731. https://doi.org/10.1111/j.1365-2214.2006.00689.x

Kaplan, B., Wilson, B. N., Dewey, D., \& Crawford, S. G. (1998). DCD may not be a discrete disorder. Revista Human Movement Science, 17(4-5), 471-490.

Kirby, A., Sugden, D., \& Purcell, C. (2014). Diagnosing developmental coordination disorders. Arch Dis Child, 99(3), 292-296. https://pubmed.ncbi.nlm.nih.gov/24255567/

Jastak, S., \& Wilkinson, G. S. (1984). The Wide Range Achievement Test-Revised. Jastak Associates, Wilmington, DE, USA.

Levy, F., \& Hay, D. (2001). (Eds.) Attention, genes and ADHD. East Sussex: Brunner-Routledge.

Lingam, R., Hunt, L., Golding, J., Jongmans, M., \& Emond, A. (2009). Prevalence of Developmental Coordination Disorder using the DSM-IV at 7 years of age UK populations-based study. Pediatrics, 123, 693-700. https://doi.org/10.1542/peds.2008-1770

Lin, B. G. (1992). The Composite Speech/Language Test for school-aged children (6-15 years old). Taipei: Chinese Behavioral Science Corporation (in Chinese). 
Lin, B. G., \& Lin, M. S. (1996). The composite speech/language test for preschoolers (3-5 years, 11 months old). Taipei: Chinese Behavioral Science Corporation (in Chinese).

Losse, A., Henderson, S. E., Elliman, D., Hall, D., Knight, E., \& Jongmans, M. (1991). Clumsiness in children-do they grow out of t? a 10-year follow-up study. Revista Developmental Medicine \& Child Neurology, 33, 55-68.

Lu, L., \& Liu, H. H. (2005). Chinese Version of Peabody Picture Vocabulary Test-revised manual. (2nd ed.). Taipei: Psychological PublishingCo, Ltd.

Martin, N. C., Piek, Jan., Baynam, G., Levy, F., \& Hay, D. (2010). An examination of the relationship between movement problems and four common developmental disorders. Human Movement Science, 29(5), 799-808. https://doi.org/10.1016/j.humov.2009.09.005

Missiuna, C. (2003). Children with Developmental Coordination Disorder: at home, at school, and in the community. Can Child, Center for Childhood Disability Research, McMaster University.

Missiuna, C., Gaines, R., Soucie, H., \& McLean, J. (2006). Parents questions about developmental coordination disorder: A synopsis of curret evidence. Paedriatrics \& Child Health, 11(8), 507-512. https://doi.org/10.1093/pch/11.8.507

Missiuna, C., Moll, S., King, S., King, G., \& Law M. (2007). A trajectory of Troubles: Parent's Impressions of the Impact of Developmental Coordination Disorder. Physical \& Occupational Therapy in Pediatrics, 27(1), 81-101. https://pubmed.ncbi.nlm.nih.gov/17298942/

Papalia, D. E., \& Feldman, R. D. (2013). Desenvolvimento Humano. (12a ed.). AMGH.

Piek, J. P., Pitcher, T. M., \& Hay, D. A. (1999). Motor coordination and Kinaesthesis in boys with attention deficit-hyperactivity disorder. Revista Developmental Medicine \& Child Neurology, 41, 159-165.

Pike, Gary. R., \& Kuh, G. D. (2005). First-and second-generation college students: A comparison of their engagement and intellectual development. The Journal of Higher Education, 76(3), 276-300.

Pulzi, W., \& Rodrigues, G. M. (2015). Transtorno do Desenvolvimento da Coordenação: Uma Revisão De Literatura. Brasileira de Educação Especial, 21(3), 433-44.

Rintala, P., Pienimaki, K., Ahonen, T., Cantell, M., \& Kooistra, L. (1998). The effects of a psychomotor training programme on motor skill developmental language disorder. Revista Human Movement Science, Amsterdam, 17, 721-737.

Rodrigues, D. (2006). Dez ideias (mal) feitas sobre a educação inclusiva. In: Rodrigues, D. (Org.), Inclusão e Educação: dez olhares sobre a educação inclusiva. São Paulo: Summus.

Rosner, J., \& Simon, D. P. (1971). The auditory analysis test: an initial report. Journal of Learning Disabilities, 4, 384 - 392.

Rosner, J. (1993). Helping Children Overcome Learning Difficulties. (3a ed.). Walker and Company.

Rotta, N. T., Ohlweiler, L., \& Riesgo, R. S. (2006). Transtornos da aprendizagem: abordagem neurobiológica e multidisciplinar. Artmed.

Sartori, G., Job, R., \& Tressoldi, P. E. (2007). DDE2-Batteria per la valutazione della dislessia e disortografia evolutiva [Battery for the assessment of developmental dyslexia and dysorthographia. Firenze, Italy: Giunti, Organizzazioni Speciali.

Siqueira, C. M., \& Giannetti, J. G. (2011). Mau desempenho escolar: uma visão atual. Rev. Assoc. Med. Brasileira, 57(1), 78-87.

Schaffer, R., Law, M., Polatajko, H., \& Miller, J. (1989). A study of children with learning disabilities and sensorimotor problems or: let's not throw the baby out with the batwater. Physical and Occupation Therapy in Pediatrics, 9(3), 101-117. https://pubmed.ncbi.nlm.nih.gov/15772037/

Schoemaker, M. M., \& Kalverboer, A. F. (1994). Social and Affective Problems of Children Who are Clumsy - How Early Do They Begin? Adapted physical activity quarterly: $A P A Q, 11(2), 130-140$.

Tal-Saban, M., Zarka, S., Itamar, G., Ornoy, A., \& Parusk, S. (2012). The functional profile of young adults with suspected Developmental Coordination Disorder (DCD), Research in Developmental Disabilities, 33(6), 2193-202. https://doi.org/10.1016/j.ridd.2012.06.005

Thomas, J. R., \& Nelson, J. K. (2002). Pesquisa descritiva. Métodos de pesquisa em atividade física. (3a ed.). Porto Alegre: Artmed.

Villa, G. M., Barriopedro, M., \& Ruiz, L. M. (2020). Motor competence difficulties and attention de deficit and hyperactivity disorder (ADHD) among secondary students. Cuadernos de Psicologia del Deporte, 20(2), 47-62. https://doi.org/10.6018/cpd.360491

Visser, J. (2003). Developmental coordination disorder: a review of research on subtypes and comorbidities. Revista Humam Movement Science, 22(4-5), 479493.

Whittemore, R., \& Knafl, K. (2005). The integrative review: updated methodology. Journal of advanced nursing, 52(5), 546-53. https://pubmed.ncbi.nlm.nih.gov/16268861/

Willcutt, E. G., Boada, R., Riddle, M. W. Chhabildas, N., DeFries, J. C., \& Pennington, B. F. (2011). Colorado Learning Difficulties Questionnaire: Validation of a parent-report screening measure, Psychol assess. 23(3), 778-791. https://pubmed.ncbi.nlm.nih.gov/21574721/

Woodcock, R. W., \& Johnson, M. B. (1989). Woodcock-Johnson Psychoeducational Battery-Revised. DLM Teaching Resources, Allen, TX, USA.

Zwicker, J. G., Harris, S. R., \& Klassen, A. F. (2012). Quality of live domains affected in children with developmental coordination disorder: a review and update. Child, Care Health and Development. 39(4), 562-580. https://pubmed.ncbi.nlm.nih.gov/22515477/ 